\title{
Diagnósticos fisioterapêuticos cinético-funcionais no cuidado à saúde da mulher: revisão integrativa
}

\author{
Kinetic-physiotherapy diagnotics in women's health care: integrative review \\ Diagnóstico cinético-funcional en la atención a la salud de la mujer: revisión integradora
}

Recebido: 29/11/2021 | Revisado: 07/12/2021 | Aceito: 08/12/2021 | Publicado: 16/12/2021

Hemilly Macena de Oliveira Silva

ORCID: https://orcid.org/0000-0002-1934-0203 Centro Universitário Mário Pontes Jucá, Brasil

E-mail: macenahemilly@gmail.com

Ailton Mota do Nascimento Galvão

ORCID: https://orcid.org/0000-0002-9268-6921

Centro Universitário Mário Pontes Jucá, Brasil

E-mail: ailton_fisioterapeuta@ hotmail.com

Ahyas Sydcley Santos Alves

ORCID: https://orcid.org/0000-0001-7103-8389

Centro Universitário Mário Pontes Jucá, Brasil

E-mail: contato@ahyas.com.br

\begin{abstract}
Resumo
O estudo objetivou discutir e analisar acerca dos diagnósticos fisioterapêuticos cinético-funcionais no cuidado à saúde da mulher através de revisão integrativa. As buscas foram realizadas no portal BVS e no portal PubMed com as estratégias de busca e os descritores fisioterapia, diagnóstico, saúde da mulher e avaliação. Para o processo de extração dos dados e informações dos artigos após a leitura na íntegra, utilizou-se um instrumento de fichamentos auto-criado para esta revisão. Resultaram-se 8 termos de busca que resultaram em 147 achados, e, com os critérios de inclusão e exclusão desta revisão, foram elegidos 16 artigos. Evidenciou-se que são diversos os diagnósticos cinético-funcionais e seus contextos de aplicação no cuidado fisioterapêutico à saúde da mulher e que sua contribuição está na prática para potencializar as informações das condições de saúde e o estado funcional desses indivíduos. Indicam-se estudos futuros que sejam realizados abordando maiores especificidades da temática, como por exemplo as dos diagnósticos cinéticofuncionais na à saúde da mulher na atenção primária. Este diagnóstico cinético-funcional, na perspectiva da sistematização, se mostra uma ferramenta na contribuição e importância da avaliação, intervenção e alta fisioterapêutica nos cuidados a mulher.
\end{abstract}

Palavras-chave: Diagnóstico; Especialidade de fisioterapia; Saúde da mulher; Avaliação em saúde.

\begin{abstract}
The study aimed to discuss and analyze the kinetic-functional physiotherapeutic diagnoses in women's health care through an integrative review. The search was made in the VHL and PubMed portal with the search strategies and the descriptors physiotherapy, diagnosis, women's health, and evaluation. For the process of extracting data and information from the articles after reading them in their entirety, a self-created fiching instrument was used for this review. Eight search terms resulted in 147 findings, and with the inclusion and exclusion criteria of this review, 16 articles were chosen. It was evident that there are several kinetic-functional diagnoses and their contexts of application in physiotherapeutic care to women's health, and that their contribution is in the practice to enhance the information of health conditions and functional status of these individuals. It is recommended that future studies be conducted addressing greater specificities of the theme, such as kinetic-functional diagnosis in women's health in primary care. This kinetic-functional diagnosis, from the perspective of systematization, is a tool to contribute to the importance of assessment, intervention and physiotherapeutic.
\end{abstract}

Keywords: Diagnosis; Physical therapy specialty; Women's health; Health Assessment.

\section{Resumen}

El estudio tuvo como objetivo discutir y analizar los diagnósticos fisioterapéuticos cinético-funcionales en el cuidado de la salud de la mujer a través de una revisión integradora. Las búsquedas se realizaron en la BVS y en PubMed con las estrategias de búsqueda y los descriptores fisioterapia, diagnóstico, salud de la mujer y evaluación. Para el proceso de extracción de datos e información de los artículos después de leerlos en su totalidad, se utilizó un instrumento de ficha de creación propia para esta revisión. Ocho términos de búsqueda dieron lugar a 147 hallazgos y, con los criterios de inclusión y exclusión de esta revisión, se eligieron 16 artículos. Se evidenció que existen varios diagnósticos cinéticofuncionales y sus contextos de aplicación en la atención fisioterapéutica a la salud de la mujer y que su contribución 
está en la práctica para mejorar la información de las condiciones de salud y el estado funcional de estos individuos. Se indica la realización de futuros estudios que aborden mayores especificidades del tema, como los diagnósticos cinéticofuncionales en la salud de la mujer en atención primaria. Este diagnóstico cinético-funcional, en la perspectiva de la sistematización, es una herramienta en la contribución e importancia de la evaluación, intervención y alta fisioterapéutica.

Palabras clave: Diagnóstico; Especialidad de fisioterapia; Salud de la mujer; Evaluación de la salud.

\section{Introdução}

O Conselho Regional de Fisioterapia e Terapia Ocupacional da primeira região (Crefito-1), transpondo definição do Conselho Federal de Fisioterapia e Terapia Ocupacional (COFFITO), traz que que a Fisioterapia em sua ciência objetiva a avaliação, prevenção e cuidado principalmente a distúrbios da cinesia humana com o movimento humano como objeto de estudo essencial (Crefito-1, 2021).

O Conselho Regional de Fisioterapia e Terapia Ocupacional da quarta região (Crefito-4) contribui com esta definição ao trazer que, entre outros mecanismos próprios, as ações fisioterapêuticas se fundamentam no estudo da cinesiologia, da biomecânica e da sinergia funcional (Crefito-4, 2021).

Segundo a OMS (Organização Mundial de Saúde, 2015), desde a atenção básica, à atenção ambulatorial, a atenção nas urgências e emergências até a atenção hospitalar, a Fisioterapia tem a funcionalidade humana como seu principal foco de trabalho. A Classificação Internacional de Funcionalidade Humana (CIF) define a funcionalidade humana como a junção das funções humanas em suas totalidades (corpo, atividades e participação) para a análise das incapacidades humanas, como as limitações, restrições e deficiências (OMS, 2015).

O profissional fisioterapeuta, segundo o CREFITO-4 (2021), é habilitado para a realização de diagnóstico específico da categoria, o diagnóstico cinético-funcional (diagnósticos dos distúrbios cinético-funcionais). Este diagnóstico é de suma importância para guiar suas ações e práticas desde a avaliação, a prescrição, as intervenções, o prognóstico e a alta. Uma das áreas de atuação da Fisioterapia é na saúde da mulher. Esta especialidade profissional foi disciplinada por meio da Resolução COFFITO 401, de 18 de agosto de 2011 (Souza \& Nicida, 2019).

Souza e Nicida põem também que enquanto regulamentação ainda é uma área de atuação recente mas que já vem de uma prática secular nos serviços de saúde, da atenção básica à hospitalar. O cuidado fisioterapêutico nessa área, segundo o Ministério da Saúde (2019), implica basear-se nas necessidades físicas, mentais e sociais desse público e prestar uma abordagem integral através de ações de promoção da saúde, prevenção de riscos e agravos de saúde, tratamento adequado aos problemas do início da vida sexual reprodutiva, durante a gestação, parto e puerpério, até o período de climatério e menopausa. O Protocolo de Assistência à Saúde da Mulher na Atenção Primária (2021), traz que além das ações relatadas anteriormente, também é objetivo de cuidado do fisioterapeuta o diagnóstico cinético-funcional nestes problemas baseando-se na abordagem das funcionalidades à mulher em todos os seus ciclos vitais.

O fisioterapeuta no cuidado à saúde da mulher deve seguir as preconizações da política nacional a este público e não deve atuar de forma simplista, restringida e segmentada, direcionando suas condutas para além do âmbito do ciclo gravídicopuerperal (Morais; Luiz, 2018).

Este cuidado deve pontuar também a saúde sexual e reprodutiva, com ações e condutas com ênfase em atividades preventivas sem se limitar a fases de vida nas quais a mulher tem capacidade de reprodução, mas em todo o seu ciclo de vida (Morais \& Luiz, 2018). A atuação do fisioterapeuta à mulher visa promover, entre outras ações, a prevenção de complicações, desconfortos e disfunções musculoesqueléticas, sexuais e uroginecológicas, o alívio das dores, as orientações posturais e a percepção corporal (Souza \& Nicida, 2019). 
Esse cuidado visa também as orientações para amamentação, cuidado nas incontinências (urinária e fecal) e outros comprometimentos de funções uroginecológicas (Souza \& Nicida, 2019), orientações nas atividades de vida diária (AVD's) e na promoção de qualidade de vida deste público (Santos et al., 2017).

Esse cuidado deve ter como eixos prioritários: os relativos a saúde sexual e

reprodutiva, os quais objetivam a integralidade da assistência sob o enfoque de gênero; a humanização do atendimento; a atenção em planejamento reprodutivo; o combate à violência doméstica e sexual; a prevenção e o tratamento das doenças sexualmente transmissíveis, câncer de colo e mama. As atividades clínicas devem considerar as condutas para promoção, prevenção, proteção e recuperação da saúde, sob uma abordagem transdisciplinar (Xavier et al., 2015, p. 2).

Neste cenário, estudos trazem à tona a relação entre avaliação e intervenção fisioterapêutica neste âmbito de cuidado à saúde da mulher numa visão de função, independência e funcionalidade em todos os seus ciclos de vida (Burti et al., 2016), e trazem também, a importância da realização das avaliações funcionais para o fortalecimento da prática profissional pelo fisioterapeuta (Melo et al., 2021).

Pelo contexto apresentado, faz-se necessária a discussão acerca da atuação do fisioterapeuta na saúde da mulher enquanto seu papel nos diagnósticos cinético-funcionais.

\section{Metodologia}

Este estudo trata-se de um estudo de pesquisa bibliográfica, que segundo Garcia (2016) é aquele que se realiza com base em material já elaborado, constituído principalmente de livros, artigos científicos e outros escritos. Embora em quase todos os estudos seja exigido algum tipo de trabalho dessa natureza, há pesquisas desenvolvidas exclusivamente a partir de fontes bibliográficas. Nela, o pesquisador deve propor um problema de pesquisa e um objetivo que estejam em consonância e que a resposta que será buscada está nos livros, artigos, teses, dissertações e ainda, com o advento da internet, muitos dados poderão ser buscados na rede, ou ainda, a resposta encontrada seja o contrário do que está nos livros e artigos (p. 292).

Adotou-se a abordagem qualitativa, descritiva e analítica através de revisão sistemática integrativa, que seguiu as orientações de elaboração e explicação do Relatório de Preferências para Revisões Sistemáticas e Metanálise (Galvão et al., 2015).

Esta revisão também teve como objetivos específicos: Conceituar, definir e discutir o diagnóstico fisioterapêutico cinético-funcional no cuidado à saúde da mulher; Identificar os principais diagnósticos fisioterapêuticos cinético-funcionais neste cuidado; Analisar a importância e contribuições desse diagnósticos para o cuidado fisioterapêutico a saúde da mulher e a práxis fisioterapêutica; Analisar os contextos, fragilidades e potencialidade desta prática; e, Levantar e analisar as lacunas teóricas e perspectivas desta temática.

A revisão deste estudo realizou-se entre 13 de setembro de 2021 a 28 de novembro de 2021, guiando-se pelas literaturas de normatizações de revisões sistemáticas integrativas (Einstein, 2010), e, seguiu as respectivas etapas: 1) definição da problemática, das fontes de dados, das estratégias de busca e dos critérios de inclusão e exclusão; 2) busca com as estratégias nas bases de dados; 3) identificação e organização da amostragem selecionada; 4) avaliação crítica, categorização e integração das evidências; 5) interpretação, análise descritiva e discussão dos resultados; e 6) escrita e apresentação do conhecimento produzido.

Por serem fontes de dados mais acessíveis e adequadas ao tipo e tempo de realização deste estudo, optou-se pela utilização de portais e bases de dados eletrônicas como fontes para as buscas da literatura, as quais foram: o portal BVS (Biblioteca Virtual em Saúde) e o portal PubMed. Optou-se por essas bases de dados por serem portais reúnem diversas outras bases de dados em seus resultados contendo as principais publicações nacionais e internacionais da área da saúde. 
Como estratégia de busca, foram utilizados inicialmente os operadores booleanos AND, OR e NOT associados às estratégias de trucagem, as interfaces dos descritores no singular, plural, entre aspas, e, os descritores em português, espanhol e inglês. Os descritores selecionados para esta pesquisa que representaram a problemática no portal Descritores em Ciências da Saúde (DECS, 2021) foram: fisioterapia, diagnóstico, saúde da mulher e avaliação.

Para serem incluídos nesta revisão adotou-se que os estudos deveriam: serem artigos científicos dos portais, nacionais e/ou internacionais; representar o objetivo, problema da pesquisa e descritores no título, resumo, no assunto, palavras chaves e/ou texto completo; serem publicados de 2016 a 2021; estarem no português, inglês e/ou espanhol; serem pesquisas bibliográficas e/ou pesquisas empíricas (experimentais/primárias); e serem publicações completas.

Excluíram-se desta revisão aqueles resultados que se enquadraram nos critérios de inclusão, mas que estivessem duplicados nas bases de dados; fossem livros, editoriais e/ou publicações governamentais; e, não estivessem disponíveis (artigos pagos).

Os artigos que resultaram das aplicações dos critérios, para serem selecionados e elegidos nesta revisão foram aqueles que após a leitura do título, resumo e seu texto na íntegra representaram o objetivo da pesquisa e/ou alguma abordagem da temática que gerasse discussão para esta pesquisa. Para o processo de extração dos dados e informações dos artigos após a leitura na íntegra, utilizou-se um instrumento de fichamentos auto-criado para esta revisão. O instrumento de fichamento foi executado em quadros com a análise crítica e individual de cada artigo com a coleta de dados de identificação como título, autores, etc, e, dados conceituais dos artigos, como os principais resultados, procedimento e o que o artigo abordava referente à problemática desta revisão que contribuísse para a mesma).

A revisão e as análises deste estudo realizaram-se numa lógica crítica, comparativa, discursiva e descritiva dos achados em cada etapa através da observação, contagem numérica, descrição e classificação (esta última, o processo de ordenamento e organização das ideias, através da tipificação/categorização, a priori e posteriori, escolhendo e criando-se as categorias de temas para as discussões que emergiram no decorrer da leitura e fichamento. A revisão e análise foram realizadas por dois revisores de forma independente e em consenso nos pontos de divergências.

\section{Resultados}

Dos artigos elegidos, foram encontradas diversas características de diversidade, como o local de realização, a temática principal, os métodos e procedimentos e os desfechos encontrados. Dentre os principais achados, observou-se o que esses artigos abordavam sobre a temática desta revisão e o que contribuam para a discussão acerca dos diagnósticos fisioterapêuticos cinéticofuncionais no cuidado à saúde da mulher em seus diversos contextos. No quadro trazido logo abaixo, o quadro 1, apresenta-se a síntese das informações quanto aos dados de identificação e as principais contribuições dos artigos para a discussão desta revisão, numa matriz.

Quadro 1. Informações de identificação e principais contribuições.

\begin{tabular}{|c|c|c|}
\hline Título do artigo (em português) & Autores e ano de publicação do estudo & Principais contribuições para a discussão desta revisão \\
\hline $\begin{array}{ll}\text { 1. AVALIAÇÃO } & \text { DO } \\
\text { DESEMPENHO FUNCIONAL } & \text { DE } \\
\text { MULHERES SUBMETIDAS AO } & \text { AO } \\
\text { TRATAMENTO CIRÚRGICO PARA O } \\
\text { CÂNCER DE MAMA }\end{array}$ & $\begin{array}{l}\text { MARIANA } \quad \text { TIROLLI } r \text { RETT } \\
\text { BERGAMASCO, } \\
\text { LESSA, } 2020 .\end{array}$ & $\begin{array}{l}\text { Traz discussões do cuidado à mulher em tratamento de câncer de } \\
\text { mama sobre avaliação e diagnóstico fisioterapêutico quanto a } \\
\text { funções de dor, desempenho funcional e qualidade de vida; traz } \\
\text { escalas e medidas que a partir de seus resultados, podem ser usadas } \\
\text { para a instituição dos diagnósticos cinéticos- funcionais. }\end{array}$ \\
\hline $\begin{array}{lrr}2 . & \text { ANÁLISE DA FORÇA } & \text { DOS } \\
\text { MÚSCULOS DO ASSOALHO PÉLVICO } \\
\text { EM } \quad \text { MULHERES } & \text { COM } \\
\text { INCONTINÊNCIA URINÁRIA } & \text { DE }\end{array}$ & 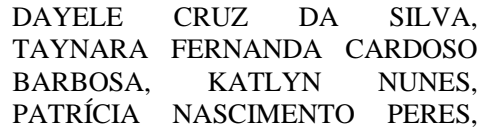 & $\begin{array}{l}\text { Traz discussões do cuidado à mulher com incontinência urinária de } \\
\text { esforço sobre avaliação e diagnóstico fisioterapêutico quanto a } \\
\text { funções de força do assoalho pélvico; traz escalas e medidas que a } \\
\text { partir de seus resultados, podem ser usadas para a instituição dos }\end{array}$ \\
\hline
\end{tabular}




\begin{tabular}{|c|c|c|}
\hline ESFORÇO & $\begin{array}{l}\text { DANIELA SALDANHA WITTIG, } \\
\text { KELLEY CRISTINA COELHO, } 2017 .\end{array}$ & diagnósticos cinéticos- funcionais. \\
\hline $\begin{array}{l}\text { 3. ROTINAS DE UM SERVIÇO DE } \\
\text { FISIOTERAPIA EM SAÚDE DA } \\
\text { MULHER EM UM CENTRO } \\
\text { OBSTÉTRICO }\end{array}$ & $\begin{array}{l}\text { DANIELLE SANTANA MACÊDO } \\
\text { SODRÉ, ALINE MANTA DA SILVA1, } \\
\text { ANA CLAUDIA MACHADO PEREIRA } \\
\text { E SILVA, KEITH FRÓES ORRICO, } \\
2019 .\end{array}$ & $\begin{array}{l}\text { Traz exemplos de avaliação funcional obstétrica que a partir de seus } \\
\text { resultados, podem ser usados para a instituição dos diagnósticos } \\
\text { cinéticos- funcionais. }\end{array}$ \\
\hline $\begin{array}{l}\text { 4. PROPOSTA DE PROTOCOLO } \\
\text { DE AVALIAÇÃO E TREINAMENTO } \\
\text { DA MUSCULATURA DO ASSOALHO } \\
\text { PÉLVICO PARA ATENDIMENTO À } \\
\text { MULHER COM INCONTINÊNCIA } \\
\text { URINÁRIA }\end{array}$ & $\begin{array}{l}\text { GISELA MARIA ASSIS, CAMILLA } \\
\text { PINHEIRO CRISTALDI DA SILVA, } \\
\text { GISELE MARTINS, 2021. }\end{array}$ & $\begin{array}{l}\text { Traz discussões do cuidado à mulher com incontinência urinária } \\
\text { sobre avaliação e diagnóstico fisioterapêutico quanto a funções de } \\
\text { força do assoalho pélvico; traz escalas e medidas que a partir de seus } \\
\text { resultados, podem ser usadas para a instituição dos diagnósticos } \\
\text { cinéticos- funcionais. }\end{array}$ \\
\hline $\begin{array}{l}\text { 5. CORRELAÇÃO ENTRE A } \\
\text { LOMBALGIA CROONICA E A FUNÇÃO } \\
\text { SEXUAL FEMININA }\end{array}$ & \begin{tabular}{lrr} 
HELOISA & \multicolumn{2}{r}{ FLORENTINO } \\
DRUMMOND, & THIAGO & SAIKALI \\
FARCIC, NELSON CARVAS JUNIOR, \\
CRISTIANO & SCHIAVINATO \\
BALDAN, & IGOR & FAGIOLI \\
BORDELLO & MASSON, & ALINE, \\
FERNANDA PEREZ MACHADO, 2020.
\end{tabular} & $\begin{array}{l}\text { Traz discussões do cuidado à mulher com dor lombar e a função } \\
\text { sexual comprometida sobre avaliação e diagnóstico fisioterapêutico } \\
\text { quanto a funções de força do assoalho pélvico; traz escalas e } \\
\text { medidas que a partir de seus resultados, podem ser usadas para a } \\
\text { instituição dos diagnósticos cinéticos- funcionais. }\end{array}$ \\
\hline $\begin{array}{lcr}\text { 6. MASSAGEM } & \text { PERINEAL } \\
\text { MELHORA } & \text { A } & \text { DISPAREUNIA } \\
\text { CAUSADA POR } & \text { TENSÃO DOS } \\
\text { MÚSCULOS DO ASSOALHO PÉLVICO }\end{array}$ & $\begin{array}{l}\text { ANA PAULA MOREIRA DA SILVA, } \\
\text { MARY LOURDES MONTENEGRO, } \\
\text { MARIA BEATRIZ FERREIRA } \\
\text { GURIAN, ANDREIA MOREIRA DE } \\
\text { SOUZA MITIDIERI, LUCIA ALVES } \\
\text { DA SILVA LARA } \\
\text { BENEDICTO POLI-NETO, JURO } \\
\text { CESAR ROSA E SILVA, 2017. }\end{array}$ & $\begin{array}{l}\text { Traz discussões do cuidado à mulher com dispareunia sobre } \\
\text { avaliação e diagnóstico fisioterapêutico quanto a funções de tensão } \\
\text { e força do assoalho pélvico; traz escalas e medidas que a partir de } \\
\text { seus resultados, podem ser usadas para a instituição dos } \\
\text { diagnósticos cinéticos- funcionais. }\end{array}$ \\
\hline $\begin{array}{l}7 . \quad \text { TRATAMENTO } \\
\text { MANIPULATIVO OSTEOPÁTICO } \\
\text { MUSCULOESQUELÉTICO } r \text { EM } \\
\text { MULHERES COM INCONTINÊNCIA } \\
\text { URINÁRIA NÃO COMPLICADA }\end{array}$ & $\begin{array}{l}\text { LILIAN DE CASTRO PONZONI, } \\
\text { ERICKA KIRSTHINE VALENTIN, } \\
\text { FABRÍCIO BORGES CARRERETTE, } \\
\text { RONALDO DAMIÃO, } 2019 .\end{array}$ & $\begin{array}{l}\text { Traz discussões do cuidado à mulher com incontinência urinária não } \\
\text { complicada sobre avaliação e diagnóstico fisioterapêutico quanto a } \\
\text { funções de força do assoalho pélvico; traz escalas e medidas que a } \\
\text { partir de seus resultados, podem ser usadas para a instituição dos } \\
\text { diagnósticos cinéticos- funcionais. }\end{array}$ \\
\hline $\begin{array}{l}8 . \quad \text { CUIDADO } \\
\text { FISIOTERAPÊUTICO NA FUNÇÃO } \\
\text { SEXUAL FEMININA: INTERVENÇÃO } \\
\text { EDUCATIVA NA MUSCULATURA DO } \\
\text { ASSOALHO PÉLVICO }\end{array}$ & $\begin{array}{l}\text { CHRISTIANE } \text { KELEN LUCENA DA } \\
\text { COSTA, MARIA } \text { HELENA } \\
\text { CONSTANTINO SPYRIDES, ANA } \\
\text { CRISTINA DA NÓBREGA MARINHO, } \\
\text { MARIA BERNARDETE CORDEIRO } \\
\text { DE SOUSA, 2018. }\end{array}$ & $\begin{array}{l}\text { Traz discussões do cuidado à mulher quanto sua função sexual sobre } \\
\text { avaliação e diagnóstico fisioterapêutico quanto a funções de força } \\
\text { do assoalho pélvico; traz escalas e medidas que a partir de seus } \\
\text { resultados, podem ser usadas para a instituição dos diagnósticos } \\
\text { cinéticos- funcionais. }\end{array}$ \\
\hline $\begin{array}{l}\text { 9. PERFIL CLÍNICO E CINÉTICO- } \\
\text { FUNCIONAL DE PUÉRPERAS EM } \\
\text { PERÍODO IMEDIATO }\end{array}$ & $\begin{array}{l}\text { BRUNA LIMA ANDRADE LÉLIA } \\
\text { PEREIRA DOS SANTOS SILVA, } 2017 .\end{array}$ & $\begin{array}{l}\text { Traz discussões do cuidado à mulher em puerpério imediato sobre } \\
\text { avaliação e diagnóstico fisioterapêutico; traz escalas e medidas que } \\
\text { a partir de seus resultados, podem ser usadas para a instituição dos } \\
\text { diagnósticos cinéticos- funcionais. }\end{array}$ \\
\hline $\begin{array}{llll}10 . \quad \text { A } & \text { INFLUENCIA } & \text { DA } \\
\text { FISIOTERAPIA NA } & \text { RECUPERAÇÃO } \\
\text { FUNCIONAL DE } & \text { PACIENTES } & \text { EM } \\
\text { TRATAMENTO DO } & \text { CÂNCER } & \text { DE } \\
\text { MAMA } & & & \end{array}$ & $\begin{array}{l}\text { ANDRESSA DE OLIVEIRA PAMELA } \\
\text { TATIANA RODRIGUES, } 2021 .\end{array}$ & $\begin{array}{l}\text { Traz discussões do cuidado à mulher em recuperação funcional de } \\
\text { câncer de mama sobre avaliação e diagnóstico fisioterapêutico; traz } \\
\text { escalas e medidas que a partir de seus resultados, podem ser usadas } \\
\text { para a instituição dos diagnósticos cinéticos- funcionais. }\end{array}$ \\
\hline $\begin{array}{lr}\text { 11. PROPOSTA DE UMA FICHA DE } \\
\text { AVALIAÇÃO } & \text { FISIOTERAPÊUTICA } \\
\text { PARA } & \text { PACIENTES } \\
\text { MASTECTOMIZADAS } & \end{array}$ & $\begin{array}{lcr}\text { THAIS } & \text { CARVALHO } & \text { ROCHA } \\
\text { RODRIGUES, } & \text { ELIANE MARIA DE } \\
\text { CARVALHO, } & \text { CLAUDIA } & \text { FELIX } \\
\text { CHAVES } & \text { FERREIRA, } & \text { SILVANA } \\
\text { GONÇALVES } & \text { CARDOSO, JULIANA } \\
\text { CAROLINA } & \text { CAIXETA, } & \text { GIAN } \\
\text { FONSECA } & \text { DO } & \text { ROZARIO, } \\
\text { FREDERICO } & \text { TADEU } & \text { DELOROSO, } \\
2019 . & & \end{array}$ & $\begin{array}{l}\text { Traz discussões do cuidado à mulher mastectomizada sobre } \\
\text { avaliação e diagnóstico fisioterapêutico; traz escalas e medidas que } \\
\text { a partir de seus resultados, podem ser usadas para a instituição dos } \\
\text { diagnósticos cinéticos- funcionais. }\end{array}$ \\
\hline $\begin{array}{l}\text { 12. AVALIAÇÃO DA FORÇA } \\
\text { MUSCULAR DO ASSOALHO PÉLVICO } \\
\text { EM PROFISSIONAIS DO SEXO NA } \\
\text { CIDADE DE FORTALEZA/CE }\end{array}$ & $\begin{array}{l}\text { MARIA MERCÊS MIRANDA } \\
\text { BARBOSA DE SOUZA, THIAGO } \\
\text { BRASILEIRO DE VASCONCELOS, } \\
\text { JULIANA LERCHE VIEIRA ROCHA } \\
\text { PIRES, RAIMUNDA HERMELINDA }\end{array}$ & $\begin{array}{l}\text { Traz discussões do cuidado à mulher profissional do sexo sobre } \\
\text { avaliação e diagnóstico fisioterapêutico quanto a funções de força } \\
\text { do assoalho pélvico; traz escalas e medidas que a partir de seus } \\
\text { resultados, podem ser usadas para a instituição dos diagnósticos } \\
\text { cinéticos- funcionais. }\end{array}$ \\
\hline
\end{tabular}




\begin{tabular}{|c|c|c|}
\hline & $\begin{array}{l}\text { MAIA MACENA, VASCO PINHEIRO } \\
\text { DIÓGENES BASTOS, } 2016 .\end{array}$ & \\
\hline $\begin{array}{l}\text { 13. ALTERAÇÕES SEXUAIS NO } \\
\text { CLIMATÉRIO DO PONTO DE VISTA } \\
\text { CINESIOLÓGICO-FUNCIONAL: } \\
\text { REVISÃO }\end{array}$ & $\begin{array}{l}\text { SABRINA NARCIZO CAMILO, } \\
\text { CAROLINA LAZZARIM DE CONTO, } \\
\text { ERICA FEIO CARNEIRO NUNES, } \\
\text { GUSTAVO F. SUTTER LATORRE, } \\
2019 .\end{array}$ & $\begin{array}{l}\text { Traz discussões do cuidado à mulher no climatério sobre avaliação } \\
\text { e diagnóstico fisioterapêutico; traz escalas e medidas que a partir de } \\
\text { seus resultados, podem ser usadas para a instituição dos } \\
\text { diagnósticos cinéticos- funcionais. }\end{array}$ \\
\hline $\begin{array}{l}\text { 14. VALIDAÇÃO DA ESCALA } \\
\text { CURTA DE AVALIAÇÃO FUNCIONAL } \\
\text { DO DESEJO SEXUAL FEMININO }\end{array}$ & $\begin{array}{lcr}\text { GUSTAVO } & \text { FERNANDO } & \text { SUTTER } \\
\text { LATORRE, } & \text { ELISSA } & \text { BOBSIN, } \\
\text { LUCIELLE } & \text { TATIANA KIST, ERICA } \\
\text { FEIO CARNEIRO NUNES, } 2020 .\end{array}$ & $\begin{array}{l}\text { Traz discussões do cuidado à mulher quanto sua função sexual sobre } \\
\text { avaliação e diagnóstico fisioterapêutico; traz escalas e medidas que } \\
\text { a partir de seus resultados, podem ser usadas para a instituição dos } \\
\text { diagnósticos cinéticos- funcionais. }\end{array}$ \\
\hline $\begin{array}{lrr}\text { 15. FISIOTERAPIA } & \text { NA } \\
\text { ANORGASMIA FEMININA: } & \text { UMA } \\
\text { REVISÃO INTEGRATIVA1 } & \end{array}$ & $\begin{array}{l}\text { DANIELI DELEVATI PAVANELO, } \\
\text { DANIELA ZENI DREHER, } 2020 .\end{array}$ & $\begin{array}{l}\text { Traz discussões do cuidado à mulher com anorgasmia sobre } \\
\text { avaliação e diagnóstico fisioterapêutico; traz escalas e medidas que } \\
\text { a partir de seus resultados, podem ser usadas para a instituição dos } \\
\text { diagnósticos cinéticos- funcionais. }\end{array}$ \\
\hline $\begin{array}{lll}\text { 16. AVALIAÇÃO DO RISCO DE } & \text { DE RISC } \\
\text { QUEDA E E EUILÍBRIO } & \text { EM } \\
\text { MULHERES NO CLIMATÉRIO } & \end{array}$ & $\begin{array}{l}\text { PATRÍCIA FRANCIELI DE PAULA } \\
\text { XAVIER ANA PAULA NASSIF } \\
\text { TONDATO DA TRINDADE, } 2018 .\end{array}$ & $\begin{array}{l}\text { Traz discussões do cuidado à mulher no climatério em risco de } \\
\text { queda sobre avaliação e diagnóstico fisioterapêutico principalmente } \\
\text { às funções de equilíbrio; traz escalas e medidas que a partir de seus } \\
\text { resultados, podem ser usadas para a instituição dos diagnósticos } \\
\text { cinéticos- funcionais. }\end{array}$ \\
\hline
\end{tabular}

Fonte: Autores (2021).

Com os descritores e estratégias utilizados resultaram-se 8 termos de busca para as duas bases de dados escolhidas para a pesquisa. Esses termos resultaram em 147 achados nas duas bases de dados. Desses 147 achados totais, encontram-se 140 resultados na base BVS e na PubMed encontraram-se 7 resultados.

Após a pesquisa nas bases de dados, com a aplicação dos critérios de inclusão e exclusão desta revisão, foram selecionados 27 artigos para leitura na íntegra. Após a leitura na íntegra foram excluídos 11 que não apresentaram com significância para alguma contribuição resultaram então como elegidos desta revisão 16 artigos.

A figura 1, nas orientações de sistematização e elaboração do PRISMA-P, apresenta o fluxograma com os resultados em cada uma das etapas de buscas e seleções desta revisão. 
Figura 1. Fluxograma de resultados das buscas e seleções.

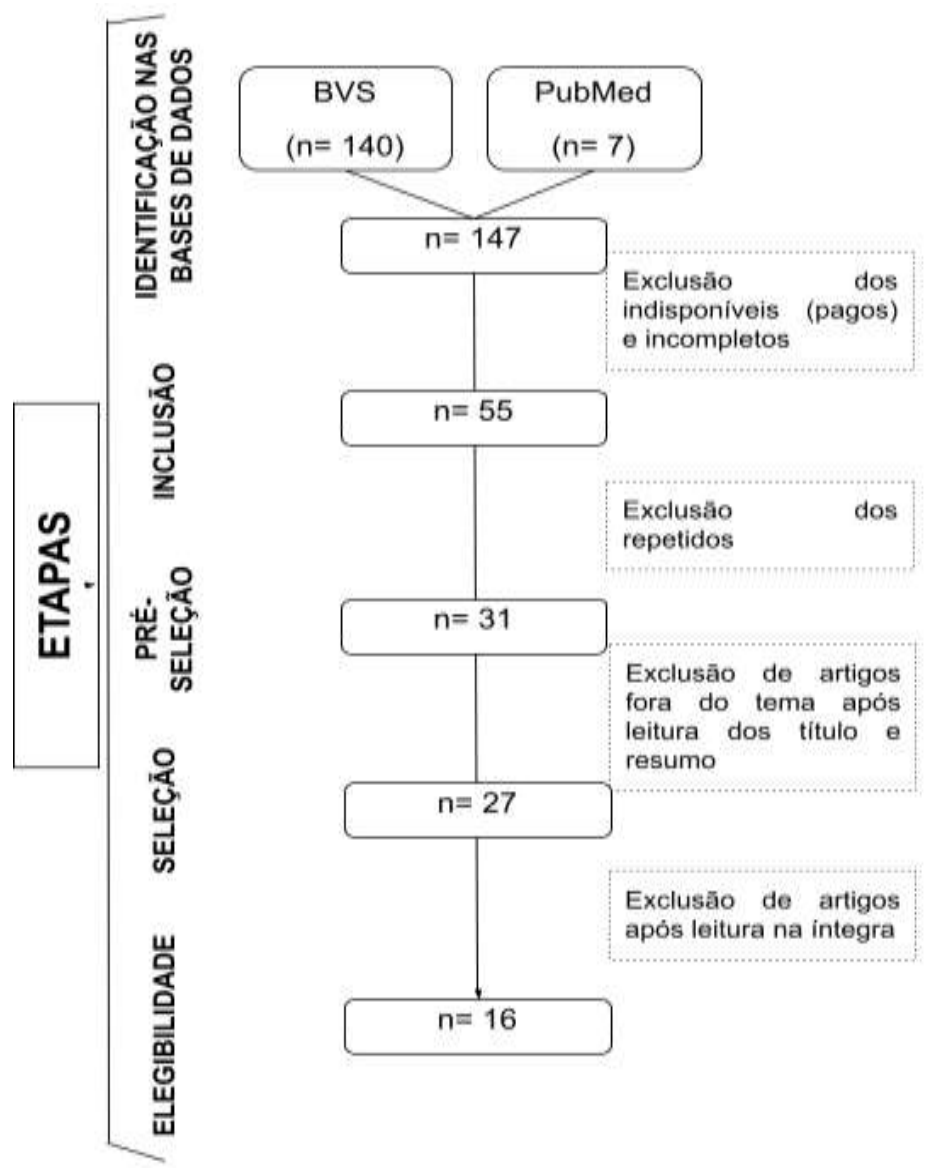

Fonte: Autores (2021).

\section{Discussão}

4.1 Conceito, definição, importância, contribuições, potencialidades e perspectivas do diagnóstico fisioterapêutico cinético-funcional no cuidado à saúde da mulher

Pode-se inferir, a partir dos resultados da integração da literatura desta revisão, sobre o conceito de diagnóstico fisioterapêutico cinético-funcional no cuidado à saúde da mulher.

a funcionalidade humana é definida como a capacidade de/para realizar atividades, como as de autocuidado até aquelas com grandes necessidades de aspectos somáticos, como força e mobilidade (Alves et al., 2019).

Sendo assim, infere-se que o diagnóstico fisioterapêutico cinético-funcional no cuidado à saúde da mulher é definido como a estratégia de cuidado avaliativo do fisioterapeuta nos aspectos cinéticos, funcionais e cinesiológicos da saúde da mulher em situação de adoecimento ou não.

Analisando o conceito trazido anteriormente, podemos inferir que o diagnóstico fisioterapêutico cinético-funcional no cuidado à saúde da mulher tem e pode apresentar diversas contribuições à prática do fisioterapeuta nesta especialidade, desde a sua avaliação, sua intervenção e a alta ao usuário.

Segundo Gazola et al. (2018) o processo de cuidado de fisioterapia aos pacientes deverá envolver a avaliação, a identificação das disfunções e das limitações funcionais associados ao problema, a definição de metas centradas no paciente, a seleção da intervenção terapêutica efetiva e a avaliação do efeito da intervenção a partir da evolução do paciente.

A avaliação no cuidado fisioterapêutico à saúde da mulher é um marco importante na construção para a construção do diagnóstico fisioterapêutico, visto que possibilitará a identificação dos objetivos do tratamento a curto, médio e longo prazos, 
assim como a prevenção à mulher, possibilitando a formulação da periodização de um programas de tratamento adequados a cada caso e agravo à saúde da mulher em seus diversos ciclos de vida (Rodrigues et al., 2019)

A sistematização das práticas dos fisioterapeuta é essencial e Gazola et al. ressaltam que estará podendo ser viabilizada pelo uso de testes padronizados para documentação do progresso do paciente antes, durante e após a intervenção. Contudo, uma prática sistematizada também inclui a elaboração de intervenções a partir da avaliação para atingir um determinado objetivo terapêutico.

Rodrigues et al. (2019) relatam que com a instituição desse diagnóstico cinético-funcional, a reprodução das reavaliações permitirão verificar a eficácia do processo de reabilitação, onde o fisioterapeuta poderá comparar os resultados quanto à cada diagnóstico com a avaliação inicial da mulher.

Frente ao dialogado, observa-se então a importância do profissional fisioterapeuta estabelecer em sua conduta diária no cuidado à mulher a prática de seu diagnóstico, devendo basear-se na cinesiologia e funcionalidade de cada usuária, em seus aspectos diversos, principalmente os obstétricos, neuromusculares, ginecológicos, sexuais, pneumofuncionais e cardiovasculares.

Visando o cuidado integral e de qualidade em todos os aspectos de saúde da mulher, a construção e definição do diagnóstico cinético-funcional de cada paciente beneficia ao fisioterapeuta poder direcionar metas e condutas específicas a cada diagnóstico, assim, potencializando seus cuidados e resultados no cuidado à saúde da mulher.

Frente ao exposto, a avaliação do fisioterapeuta especialista e atuante no cuidado à saúde da mulher é imprescindível através do diagnóstico cinético-funcional com a antecipação dos cuidados mais efetivos e a reabilitação precoce, que levarão a desfechos mais favoráveis para a mulher e seu cuidado.

Este aspecto do papel fisioterapêutico a mulher apresenta, como visto nos resultados e nessa primeira categoria de discussão, potencialidades diversas e boas perspectivas de avanço e fortalecimento à prática do fisioterapeuta.

\subsection{Principais diagnósticos fisioterapêuticos cinético-funcionais no cuidado à saúde da mulher}

Os artigos selecionados desta revisão resultaram na síntese de diversos diagnósticos cinético-funcionais do cuidado fisioterapêutico à saúde da mulher, que permeiam por diversos âmbitos do funcionamento do corpo e funções corporais da mulher, e, são resultantes das diversas formas de avaliação e instrumentos que o fisioterapeuta utiliza.

A seguir, no Quadro 2, são relatados os diagnósticos cinético-funcionais identificados, que os autores trazem em cada artigo, acerca do cuidado fisioterapêutico à saúde da mulher.

Quadro 2. Principais diagnósticos cinético-funcionais identificados no cuidado fisioterapêutico a saúde da mulher identificados.

\begin{tabular}{|c|c|}
\hline Artigo & Diagnósticos cinético-funcionais identificados \\
\hline 1 & $\begin{array}{l}\text { ALTERAÇÕES NA CICATRIZ CIRÚRGICA, ALTERAÇÃO FUNCIONAL DECORRENTE DE DOR E MEDO DE MOVIMENTAR, } \\
\text { DIFICULDADES EM REALIZAR ATIVIDADES COMUNS DO SEU COTIDIANO, COMO: VESTIR UMA BLUSA FECHADA, } \\
\text { CARREGAR UMA SACOLA PESADA OU ALCANÇAR OBJETOS EM UMA ALTURA SUPERIOR À LINHA MÉDIA DOS OMBROS, } \\
\text { ABRIR UMA TAMPA APERTADA, VIRAR UMA CHAVE, PREPARAR UMA REFEIÇÃO OU ARRUMAR SUA PRÓPRIA CAMA, } \\
\text { ALTERAÇÕES DEA AMPLITUDE DE MOVIMENTO. }\end{array}$ \\
\hline 2 & $\begin{array}{l}\text { DÉFICIT OU ALTERAÇÃO DE FORÇA E TÔNUS DOS MÚSCULOS DO ASSOALHO PÉLVICO, INCONTINÊNCIA URINÁRIA DE } \\
\text { ESFORÇO, DIFICULDADE EM REALIZAR OS TESTES NA AVALIAÇÃO, DÉFICIT DE CONHECIMENTO DA REGIÃO PÉLVICA, } \\
\text { CAPACIDADE DE RECRUTAMENTO DA MUSCULATURA, DE COORDENAÇÃO REFLEXA DURANTE O ESFORÇO, DE } \\
\text { CONSCIÊNCIA PERINEAL, ESTRATÉGIAS DE CONTROLE MOTOR ALTERADAS, PERDA DE URINA, DE CONTRAÇÃO } \\
\text { CONSCIENTE E EFETIVA, DE SUSTENTAÇÃO E DA RESISTÊNCIA URETRAL, PROLAPSO GENITAL, SENSAÇÃO DE PESO } \\
\text { PÉLVICO. }\end{array}$ \\
\hline 3 & $\begin{array}{l}\text { DÉFICIT OU ALTERAÇÃO NA POSTURA ESTÁTICA, NA BIOMECÂNICA E NA DINÂMICA CORPORAL DO PARTO, } \\
\text { ALTERAÇÕES QUE ACOMETEM O SISTEMA OSTEOMIOARTICULAR, RESPIRATÓRIO, CIRCULATÓRIO, NO PADRÃO E } \\
\text { FREQUÊNCIA RESPIRATÓRIA, NA AMPLITUDE DE MOVIMENTO ARTICULAR E FORÇA MUSCULAR DE MEMBROS, TRONCO } \\
\text { E PELVE, NO EQUILÍBRIO ESTÁTICO E DINÂMICO, NA GRADUAÇÃO DE EDEMA DEPRESSÍVEL. }\end{array}$ \\
\hline
\end{tabular}




\begin{tabular}{|c|c|}
\hline 4 & $\begin{array}{l}\text { ALTERAÇÃO DE CICATRIZES E DE PROLAPSOS EM REPOUSO, NA CONTRAÇ̃̃O DO ASSOALHO PÉLVICO, RIGIDEZ } \\
\text { MUSCULAR AO TOQUE, CONTRAÇÃO DE ASSOALHO PÉLVICO COM CONTRAÇÃO DE ABDÔMEN, GLÚTEOS E COXAS, NA } \\
\text { CAPACIDADE DE RELAXAMENTO, ESPASTICIDADE DO MÚSCULO PERINEAL, ATIVIDADE PSICOMOTORA DO MÚSCULO } \\
\text { PERINEAL PREJUDICADA, FRAQUEZA DO MÚSCULO PERINEAL, RESISTÊNCIA DO MÚSCULO PERINEAL PREJUDICADA, } \\
\text { REFLEXO DO MÚSCULO PERINEAL PREJUDICADO, HIPERATIVIDADE DO ASSOALHO PÉLVICO, PERDA URINÁRIA, PERDA } \\
\text { DE GASES OU FEZES, HESITAÇÃO DO JATO MICCIONAL, JATO FRACO OU INTERMITENTE, SENSAÇÃO DE ESVAZIAMENTO } \\
\text { INCOMPLETO, ESFORÇO EVACUATÓRIO, DOR NA RELAÇÃO SEXUAL, TENSÃO MUSCULAR E/OU RELAXAMENTO } \\
\text { INEFICAZ, AUSÊNCIA DE PROPRIOCEPÇÃO PÉLVICA, INCOORDENAÇÃO ENTRE AUMENTO DA PRESSÃO INTRA } \\
\text { ABDOMINAL E CONTRAÇÃO DA MAP. }\end{array}$ \\
\hline 5 & $\begin{array}{l}\text { DÉFICIT OU ALTERAÇÃO DE DOR LOMBAR, FUNÇÃO SEXUAL, CONSCIÊNCIA PERINEAL E FORTALECIMENTO DA } \\
\text { MUSCULATURA DO ASSOALHO PÉLVICO, NA SENSIBILIDADE VULVAR E VAGINAL, NO POSICIONAMENTO DO INDIVIIDUO } \\
\text { DURANTE O SEXO BEM COMO A SUA RESPECTIVAS ADAPTAÇÕES, NO RELAXAMENTO DOS MÚSCULOS DURANTE O } \\
\text { SEXO. }\end{array}$ \\
\hline 6 & $\begin{array}{l}\text { DÉFICIT OU ALTERAÇÃO DE DISPAREUNIA, TENSÃO DOS MÚSCULOS DO ASSOALHO PÉLVICO, NO ALÍVIO DA DOR A } \\
\text { LONGO PRAZO, INTERROMPIMENTO DA RELAÇÃO SEXUAL E EVITAR SEXO, NA DISPAREUNIA CAUSADA POR } \\
\text { SENSIBILIDADE PÉLVICA. }\end{array}$ \\
\hline 7 & $\begin{array}{l}\text { DÉFICIT OU ALTERAÇÃO EM INCONTINÊNCIA URINÁRIA NÃO COMPLICADA, NA MOBILIDADE PARA AS ARTICULAÇÕES } \\
\text { SACRO ILÍACAS, ARTICULAÇÕES DA COLUNA VERTEBRAL E ARTICULAÇÕES DOS MEMBROS INFERIORES, NA MÚSCULO } \\
\text { DIAFRAGMA, NA AMPLITUDE DE MOVIMENTO DAS ESTRUTURAS, QUADRO ÁLGICO, NA MOBILIDADE DAS } \\
\text { ARTICULAÇÕES OU DEMAIS TECIDOS CORPORAIS. }\end{array}$ \\
\hline 8 & $\begin{array}{l}\text { DÉFICIT OU ALTERAÇÃO NA FUNÇÃO SEXUAL FEMININA, MUSCULATURA DO ASSOALHO PÉLVICO, FUNÇÃO MUSCULAR } \\
\text { DO ASSOALHO PÉLVICO, INTENSIDADE, O NÚMERO DE CONTRAÇÕES, NO RELAXAMENTO PARCIAL OU MUITO LENTO, } \\
\text { NA COORDENAÇÃO INSATISFATÓRIA, NOS DOMÍNIOS DA FUNÇÃO SEXUAL: DESEJO, EXCITAÇÃO, LUBRIFICAÇÃO, } \\
\text { ORGASMO, SATISFAÇÃO E DOR. }\end{array}$ \\
\hline 9 & $\begin{array}{l}\text { DÉFICIT OU ALTERAÇÃO NA AMAMENTAÇÃO, QUADROS ÁLGICOS OU DESCONFORTO, DOR INCISIONAL, DOR POR } \\
\text { ALTERAÇÕES BIOMECÂNICAS (EM COLUNA LOMBAR) OU DOR POR ALTERAÇÕES GASTROINTESTINAIS, DOR NA REGIÃO } \\
\text { SUPRA PÚBICA, DOR NAS REGIÕES ABDOMINAL, PERINEAL, EPIGÁSTRICA, LOMBAR, CEFÁLICA E MAMÁRIA, NA } \\
\text { CONTINÊNCIA URINÁRIA, FECAL E SAUUDE SEXUAL, NA CICATRIZAÇÃO E A MELHORA DA FUNÇÃO DO ASSOALHO } \\
\text { PÉLVICO, ESTADOS DE TENSÃO, ANSIEDADE E RESISTÊNCIAS À AMAMENTAÇÃO, NA CONDIÇÃO BIOMECÂNICA DE } \\
\text { ALGUNS GRUPOS MUSCULARES, DÉFICIT NA SUSTENTAÇÃO DA MASSA VISCERAL, DISFUNÇÃO NA BIOMECÂNICA } \\
\text { RESPIRATÓRIA, NA POSTURA DO TRONCO E ESTABILIZAÇÃO DA COLUNA, ALTERAÇÁO NO RITMO GASTROINTESTINAL, } \\
\text { CONSTIPAÇÃO INTESTINAL, TRAUMAS MAMILARES E PEGA INADEQUADA DO RECÉM-NASCIDO, DIFICULDADES PARA } \\
\text { AMAMENTAR, DIÁSTASE ABDOMINAL. }\end{array}$ \\
\hline 10 & $\begin{array}{l}\text { DISFUNÇÕES MOTORAS RESULTANTES DO TRATAMENTO, NA FLEXÃO, EXTENSÃO, ABDUÇÃO, ADUÇÃO, ROTAÇÃO } \\
\text { INTERNA E EXTERNA DO OMBRO, NA PERIMETRIA DO MEMBRO SUPERIOR HIPERCIFOSE, CONTRATURA DA } \\
\text { MUSCULATURA CERVICAL, ANTERIORIZAÇÃO DA CABEÇA, ESCÁPULAS ALADAS E MAL ALINHADAS DEVIDO Ã } \\
\text { MUDANÇA BRUSCA DE PESO PELA AUSÊNCIA DA MAMA, AUMENTO DA SENSIBILIDADE NAS REGIÕES PÓSTERO } \\
\text { SUPERIORES DO MEMBRO SUPERIOR E AXILA HOMOLATERAL, ALTERAÇÕES POSTURAIS DECORRENTES DA CIRURGIA, } \\
\text { NO ALINHAMENTO HORIZONTAL DA PÉLVIS, NO ÂNGULO DO TORNOZELO, NA INCLINAÇÃO POSTERIOR DE QUADRIL E } \\
\text { ALINHAMENTO VERTICAL DO CORPO, FRAQUEZA, RIGIDEZE PARESTESIA, DIFICULDADE EM REALIZAR DETERMINADAS } \\
\text { TAREFAS, NA RECUPERAÇÃO DA MOBILIDADE FUNCIONAL. }\end{array}$ \\
\hline 11 & $\begin{array}{l}\text { DÉFICIT OU ALTERAÇÃO NA DESIDRATAÇÃO DA PELE, ADERÊNCIAS, RETRAÇÕES, PRESENÇA DE FIBROSE E } \\
\text { HEMATOMAS, PRESENÇA DO LINFEDEMA, DOR MIOFASCIAL, SENSIBILIDADE DIMINUÍDA AO TOQUE LEVE, } \\
\text { SENSIBILIDADE PROTETORA DIMINUIIDA, SENSIBILIDADE PROTETORA AUSENTE, SENSAÇÃO DE PRESSÃO PROFUNDA } \\
\text { AUSENTE, NO GRAU DE DIFICULDADE NO DESEMPENHO DE ATIVIDADES, NA AVALIAÇÃO DA FORÇA MUSCULAR DE } \\
\text { OMBRO. }\end{array}$ \\
\hline 12 & $\begin{array}{l}\text { ALTERAÇÃO NO PRAZER SEXUAL E NO ORGASMOS, PERDA URINÁRIA, FECAL, FLATOS, NA SUSTENTAÇÃO MUSCULAR } \\
\text { DO ASSOALHO PÉLVICO, ADERÊNCIA, NA SENSIBILIDADE, CICATRIZES INTERNAS, DORES NA REGIÃO E DISTOPIAS NA } \\
\text { VAGINA, NO USO DA MUSCULATURA ABDOMINAL ADICIONADO COM APNEIA, NAS PAREDES VAGINAIS, ASSIMETRIA, } \\
\text { CICATRIZES, ÁREAS ATRÓFICAS OU LACERAÇÕES, NA FORÇA E A FUNCIONALIDADE DOS MAPS. }\end{array}$ \\
\hline 13 & $\begin{array}{l}\text { DISFUNÇÃO SEXUAL APÓS A MENOPAUSA, BAIXA LUBRIFICAÇÃO, DISPAREUNIA. ALTERAÇÃO NA FUNÇÃO DA } \\
\text { MUSCULATURA DO ASSOALHO PÉLVICO E A SENSIBILIDADE LOCAL, ATROFIA VAGINA, DORES DURANTE O INTERCURSO } \\
\text { SEXUAL, CONTRATURA NA MAP, DIMINUIÇÃO DA ESPESSURA EPITELIAL VAGINAL, DA DENSIDADE DA CAMADA DO } \\
\text { MÚSCULO LISO, DOS VASOS SANGUÍNEOS, DA MORFOLOGIA E DENSIDADE DAS TERMINAÇÕES NERVOSAS E DA } \\
\text { ESTRUTURA DO COLÁGENO, INCONTINÊNCIA URINÁRIA, PROLAPSOS DE ÓRGÃOS PÉLVICOS, ALTERAÇÕES NA } \\
\text { PERCEPÇÃO MUSCULAR E COORDENAÇÃO MUSCULAR, ALTERAÇÃO DO DIAFRAGMA ABDOMINAL, NOS } \\
\text { ESTABILIZADORES DOS ÓRGÃOS PÉLVICOS, NA CONTINÊNCIA URINÁRIA FECAL, NAS FASES DA RESPOSTA SEXUAL } \\
\text { FEMININA COMO DESEJO, EXCITAÇÃO E ORGASMO. }\end{array}$ \\
\hline 15 & $\begin{array}{l}\text { DÉFICIT OU ALTERAÇÃO NO GRAU DE FORÇA MUSCULAR, TEMPO TOTAL DE SUSTENTAÇÃO DA CONTRAÇÃO, NA } \\
\text { CAPACIDADE DE REPETIR ESTA CONTRAÇÃO SUSTENTADA E AINDA NA COORDENAÇÃO NECESSÁRIA PARA REALIZAR } \\
\text { CONTRAÇÕES DE FORÇA TOTAL E RELAXAMENTO COMPLETO. }\end{array}$ \\
\hline
\end{tabular}


Fonte: Autores (2021).

Observa-se a predominância dos estudos nos aspectos de diagnósticos cinéticos-funcionais no cuidado à saúde da mulher referentes à função muscular, principalmente do assoalho pélvico, e à função e mobilidade de membros superiores em pacientes no tratamento de câncer de mama.

\subsection{Contextos e instrumentos de utilização do diagnóstico cinético-funcional no cuidado fisioterapêutico à saúde da mulher}

Diversos contextos dos diagnósticos fisioterapêuticos-cinéticos foram observados nos artigos, como os contextos referentes à métodos avaliativos, faixa etária, funcionalidades do corpo, recursos, resultados, aplicações, entre outros.

Identificou-se, quantos aos instrumentos avaliativos, que podem ser utilizados para a elaboração destes diagnósticos, as fichas de avaliações, observação, palpação, escalas, testes, aparelhos e medidas gerais, específicos ou adaptados, que a partir de suas execuções e resultados pode-se estabelecer os diagnósticos fisioterapêuticos cinético-funcionais na saúde da mulher.

Foram identificados diversos contextos de atuação e instrumentos avaliativos nos artigos, que estão explanados no Quadro 3.

Quadro 3. Contextos de atuação e instrumentos avaliativos.

\begin{tabular}{|c|c|}
\hline Artigo & Contextos de atuação e instrumentos avaliativos \\
\hline 1 & $\begin{array}{l}\text { APÓS CIRURGIA DE MAMA, DOR, QUALIDADE DE VIDA, DESEMPENHO FUNCIONAL DO MEMBRO SUPERIOR HOMO E } \\
\text { BILATERAL, DASH (DESEMPENHO FUNCIONAL DO MEMBRO SUPERIOR HOMOLATERAL), TAIS COMO: COLOCAR UM } \\
\text { OBJETO EM UMA ALTURA SUPERIOR À LINHA MÉDIA DOS OMBROS, LAVAR SUA CABEÇA OU ABOTOAR UM SUTIÃ. }\end{array}$ \\
\hline 2 e 4 & $\begin{array}{l}\text { ASSOALHO PÉLVICO E INCONTINÊNCIA URINÁRIA, ESTUDO URODINÂMICO, PERINEÔMETRO E O TEMPO DE } \\
\text { MANUTENÇÃO DA CONTRAÇÃO DO MAP, AVALIAÇÃO POR MEIO DE ELETROMIOGRAFIA, PERINEOMETRIA PALPAÇÃO } \\
\text { BIDIGITAL, EXAME FÍSICO DA PELVE, ANAMNESE PRECISA FOCANDO A QUEIXA PRINCIPAL DA PACIENTE E FATORES DE } \\
\text { RISCO PARA DISFUNÇÕES DO ASSOALHO PÉLVICO. INSPEÇÃO ESTÁTICA, AVALIAR UMIDADE E ELASTICIDADE DA } \\
\text { MUCOSA VAGINAL; MANOBRA DE VALSALVA (FORÇA DE EVACUAÇO), OBSERVAR ESTABILIDADE DE INTROITO } \\
\text { VAGINAL E PROGRESSÃO DE PROLAPSOS (PROJEÇÃO DE ÓRGÃO PELO CANAL VAGINAL); TOQUE VAGINAL BIDIGITAL } \\
\text { (UNIDIGITAL SE ATROFIA COM DESCONFORTO OU ARDÊNCIA AO TOQUE), AVALIAR PROPRIOCEPÇÃO (PERCEPÇÃO DA } \\
\text { MUSCULATURA CORRETA A SER TRABALHADA); AVALIAR FORÇA MUSCULAR (PELA ESCALA DE OXFORD* EM } \\
\text { CONTRAÇÃO MÁXIMA), PERINEÔMETRO, AVALIAR CAPACIDADE DE RELAXAMENTO, AVALIAR COORDENAÇÃO: } \\
\text { CAPACIDADE DE CONTRAIR QUANDO SOLICITADA E RELAXAR QUANDO SOLICITADA OU CONTRAIR EM DIFERENTES } \\
\text { NÍVEIS DE FORÇA. }\end{array}$ \\
\hline 3 & $\begin{array}{l}\text { CENTRO OBSTÉTRICO, FUNÇÃO RESPIRATÓRIA: AVALIAR PADRÃO E FREQUÊNCIA RESPIRATÓRIA; AMPLITUDE DE } \\
\text { MOVIMENTO ARTICULAR E FORÇA MUSCULAR (TESTE MUSCULAR MANUAL) DE MEMBROS, TRONCO E PELVE, } \\
\text { EQUILÍBRIO ESTÁTICO PELO TESTE DE ROMBERG E DINÂMICO ATRAVÉS DO TIMED GET UP AND GO TEST (TUGT); O } \\
\text { SISTEMA VASCULAR-LINFÁTICO: POR INSPEÇÃO, PALPAÇÃO E GRADUAÇÃO DE EDEMA DEPRESSÍVEL (CLASSIFICAÇÃO } \\
\text { DE EDEMA DE ACORDO COM A MAGNITUDE). }\end{array}$ \\
\hline 5 & $\begin{array}{l}\text { DOR LOMBAR CRÔNICA E FUNÇÃO SEXUAL FEMININA, QUESTIONÁRIOS, O ROLAND-MORRIS DISABILITY } \\
\text { QUESTIONNAIRE E O QS-F, QUANTIFICAÇÃO DOR USANDO A ESCALA VISUAL ANALÓGICA (VAS) E FERRAMENTAS DE } \\
\text { MEDIÇÃO DE FUNCIONALIDADE, COMO O OSWESTRY DISABILITY INDEX (ODI), ÍNDICE DE FUNÇÃO SEXUAL FEMININA } \\
\text { (FSFI). }\end{array}$ \\
\hline 7 & $\begin{array}{l}\text { OSTEOPÁTICO MUSCULOESQUELÉTICO E IU NÃO COMPLICADA, AVALIAÇÃO PÉLVICA QUESTIONÁRIOS) } \\
\text { INTERNATIONAL CONSULTATION ON INCONTINENCE QUESTIONNAIRE - SHORT FORM ICIQ - UI SHORT FORM } \\
\text { QUANTIFICA E QUALIFICA A INCONTINÊNCIA URINÁRIA E AVALIA IMPACTO NA QUALIDADE DE VIDA E O STRESS } \\
\text { URINARY INCONTINENCE QUESTIONNAIRE - SUIQ - AVALIA A PREVALÊNCIA DOS SINTOMAS DE ESFORÇO E DE } \\
\text { URGÊNCIA, E O INTERNATIONAL CONSULTATION ON INCONTINENCE QUESTIONNAIRE OVERACTIVE BLADDER - OABQ } \\
\text { (ICIQ-OAB QOL) - AVALIAÇÃO DA PRESENÇA DE SINTOMAS DE BEXIGA HIPERATIVA, E O EUROQOL-5D (EQ5D) - AVALIA } \\
\text { A QUALIDADE DE VIDA E A SAÚDE GERAL DAS PARTICIPANTES, A AVALIAÇÃO FUNCIONAL DO ASSOALHO PÉLVICO } \\
\text { (AFA) UTILIZANDO A ESCALA DE OXFORD, TESTES DE MOBILIDADE PARA AS ARTICULAÇOEES SACRO ILÍACACA, } \\
\text { ARTICULAÇÕES DA COLUNA VERTEBRAL, E ARTICULAÇÕES DOS MEMBROS INFERIORES, BEM COMO AVALIAÇÃO DA }\end{array}$ \\
\hline
\end{tabular}




\begin{tabular}{|c|c|}
\hline & $\begin{array}{l}\text { FUNÇÃO DO MÚSCULO DIAFRAGMA, COM A PARTICIPANTE EM POSIÇÃO ORTOSTÁTICA, SENTADA E EM DECÚBITO } \\
\text { DORSAL. }\end{array}$ \\
\hline 8 & $\begin{array}{l}\text { FUNÇÃO SEXUAL FEMININA, PALPAÇÃO DIGITAL PELO MÉTODO PERFECT E PELOS PERINEÔMETROS PERITRON (9300+) } \\
\text { E BIOFEEDBACK PRESSÓRICO OU PERINA, ESCALA MODIFICADA DE OXFORD, QUESTIONÁRIO DO ÍNDICE DA FUNÇÃO } \\
\text { SEXUAL FEMININA (FEMALE SEXUAL FUNCTION INDEX (FSFI)), CLASSIFICAÇÃO DE DISFUNÇÃO SEXUAL FEMININA DA } \\
\text { AFUD (AMERICAN FOUNDATION FOR UROLOGIC DISEASE). }\end{array}$ \\
\hline 10 & $\begin{array}{l}\text { CÂNCER DE MAMA, AVALIAÇÃO FUNCIONAL DA TERAPIA DO CÂNCER-BRESTE (FACT-B). O FACT-B, QUESTIONÁRIO DE } \\
\text { FUNCIONALIDADE QUICK DASH. }\end{array}$ \\
\hline 11 & 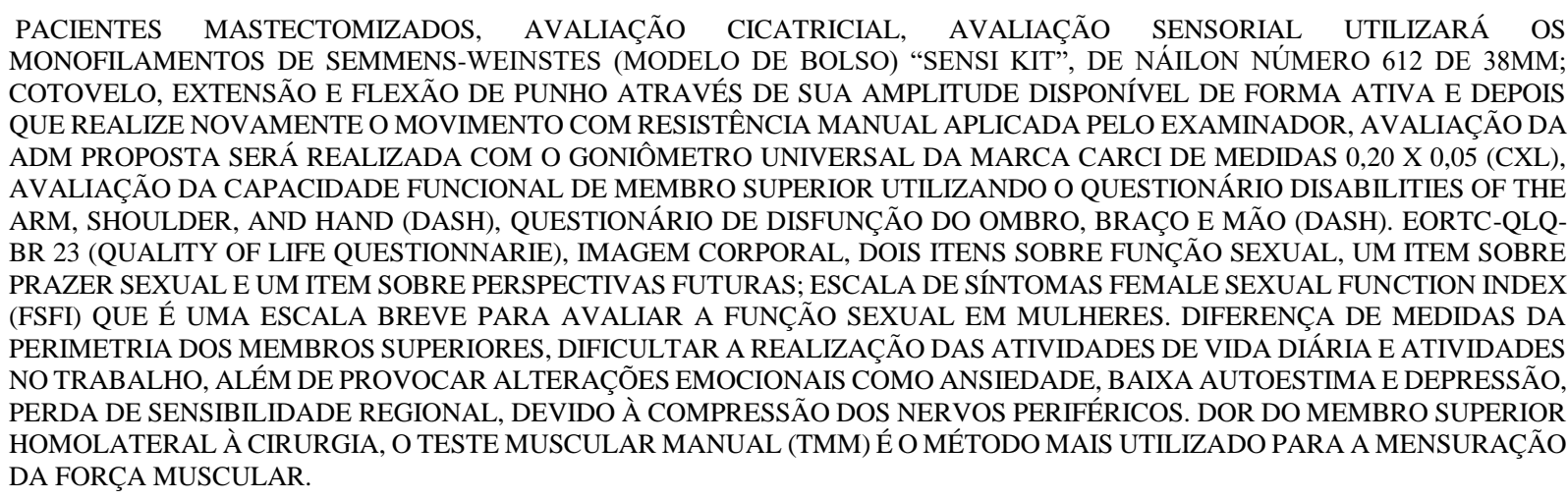 \\
\hline 12 & $\begin{array}{l}\text { FORÇA MUSCULAR DO ASSOALHO PÉLVICO EM PROFISSIONAIS DO SEXO, EXAME FÍSICO, DE INSPEÇÃO E MENSURAÇÃO } \\
\text { DA CONTRAÇÃO DOS MAP E DA AVALIAÇÃO FUNCIONAL DO ASSOALHO PÉLVICO (AFA) A PARTIR DO TOQUE BIDIGITAL } \\
\text { [11] UTILIZANDO A TABELA DE OXFORD, E PERINEÔMETRO (PERI) DE PRESSÃO [12-14] MARCA PERITRONTM } \\
\text { (AUSTRÁLIA), QUE AVALIA A PRESSÃO DA CONTRAÇÃO MUSCULAR DO ASSOALHO PÉLVICO (AP) EM CMH2O. }\end{array}$ \\
\hline 13 & CLIMATÉRIO E ALTERAÇÕES SEXUAIS, ESCALA DE OXFORD E SQ-F. \\
\hline 15 & $\begin{array}{l}\text { ANORGASMIA FEMININA, QUESTIONÁRIO DE AVALIAÇÃO E FEMALE SEXUAL FUNCTION INDEX (FSFI) QUE AVALIA: } \\
\text { DESEJO SEXUAL, EXCITAÇÃO, LUBRIFICAÇÃO, O QUESTIONÁRIO DA PERCEPÇÃO DE QUALIDADE DE VIDA (WORLD } \\
\text { HEALTH ORGANIZATION QUALITY OF LIFE - WHOQOL-BREF), ESQUEMA PERFET, QUE TEM A FINALIDADE DE AVALIAR } \\
\text { A MUSCULATURA DO ASSOALHO PÉLVICO QUANTO Â FORÇA, DURAÇÃO, REPETIÇÕES E NÚMERO DE REPETIÇÕES } \\
\text { RÁPIDAS DAS CONTRAÇÕES, PERINEÔMETROS QUE FORAM UTILIZADOS PARA VERIFICAR A MEDIDA DA PRESSÃO } \\
\text { VAGINAL. BIOFEEDBACK PRESSÓRICO, APARELHO QUE PERMITE A VISUALIZAÇÃO DA CONTRAÇÃO MUSCULAR, E QUE } \\
\text { TAMBÉM TEM SIDO UTILIZADO NA AVALIAÇÃO DO ASSOALHO PÉLVICO EM DIVERSOS ESTUDOS. }\end{array}$ \\
\hline 16 & $\begin{array}{l}\text { CLIMATÉRIO, EQUILÍBRIO E QUEDAS, A EEB É UTILIZADA COMO FERRAMENTA PARA AVALIAR O EQUILÍBRIO ESTÁTICO } \\
\text { E DINÂMICO, ÍNDICE DE TINETTI RISCO DE QUEDA. }\end{array}$ \\
\hline
\end{tabular}

Fonte: Autores (2021).

Observa-se a variedade de contextos de atuação e de instrumentos avaliativos para os aspectos de construção dos diagnósticos cinéticos-funcionais no cuidado à saúde da mulher, principalmente os referentes à função muscular, principalmente do assoalho pélvico, e à função e mobilidade.

\section{Considerações Finais}

Evidenciou-se que são diversos os diagnósticos cinético-funcionais e seus contextos de aplicação no cuidado fisioterapêutico à saúde da mulher e que sua contribuição está na prática para potencializar as informações das condições de saúde e o estado funcional desses indivíduos.

Indicam-se estudos futuros que sejam realizados abordando maiores especificidades da temática, como por exemplo as dos diagnósticos cinético-funcionais na saúde da mulher na atenção primária.

Este diagnóstico cinético-funcional, na perspectiva da sistematização, se mostra uma ferramenta na contribuição e importância da avaliação, intervenção e alta fisioterapêutica nos cuidados a mulher. 


\section{Referências}

Garcia, E. (2016). Pesquisa Bibliográfica Versus Revisão Bibliográfica - Uma Discussão Necessária. Línguas E Letras, 17(35).

Crefito 1. (2021). Conselho Regional De Fisioterapia E Terapia Ocupacional Da Primeira Região. Definição. https: Https://Www.Crefito1.Org.Br/Profissoes/Fisioterapia/.

Crefito 4. (2021). Conselho Regional De Fisioterapia E Terapia Ocupacional Da Quarta Região. Definição. https://Crefito4.Org.Br/Site/Definicao/.

Brasil. (2015). Classificação Internacional De Funcionalidade, Incapacidade E Saúde. Ministério da Saúde. Https://Portaldeboaspraticas.Iff.Fiocruz.Br/AtencaoCrianca/Classificacao-Internacional-De-Funcionalidade-Incapacidade-E-Saude-Cif/.

Coffito. 2011. Resolução N. 401/2011 - Disciplina A Especialidade Profissional De Fisioterapia Na Saúde Da Mulher E Dá Outras Providências. https: Https://Www.Coffito.Gov.Br/Nsite/?P=3164 .

Brasil. (2019). Saúde Da Mulher Na Gestação, Parto E Puerpério. Nota Técnica Para Organização Da Rede De Atenção À Saúde Com Foco Na Atenção Primária À Saúde E Na Atenção Ambulatorial Especializada. (2019). Hospital Israelita Albert Einstein: Ministério Da Saúde, 56 P.: Il.

Brasil. (2021). Protocolo Assistencial Da Saúde Da Mulher Na Atenção Primária. (2a ed.).

Freita, I. P.; \& Matias, R. H. O. (2019). Abordagem Do Fisioterapeuta No Preparo Do Assoalho Pélvico Para O Parto Natural. Uniceplac. Brasília.

Burti, et al. (2016). Assistência ao Puerpério Imediato: O Papel Da Fisioterapia. Rev Fac Ciênc Méd Sorocaba. 2016;18(4): 1938.

Melo et al. (2021). Intervenção Fisioterapêutica No Puerpério Imediato: O Que Há De Evidências Na Última Década? Research, Society And Development, $10(3)$, e 47310312849.

Bermagasco, M. T. R. \& Lessa, A. S. (2020). Avaliação Do Desempenho Funcional De Mulheres Submetidas Ao Tratamento Cirúrgico Para O Câncer De Mama. UFS.

Silva et al. (2017). Análise Da Força Dos Músculos Do Assoalho Pélvico Em Mulheres Com Incontinência Urinária De Esforço. EIPC.

Sodré et al. (2019). Rotinas De Um Serviço De Fisioterapia Em Saúde Da Mulher Em Um Centro Obstétrico. Nova fisio científica. ed 102.

Assis et al. (2021). Proposta De Protocolo De Avaliação E Treinamento Da Musculatura Do Assoalho Pélvico Para Atendimento À Mulher Com Incontinência Urinária. Rev. esc. enferm. USP 55.

Drumond et al. (2020). Correlação Entre A Lombalgia Crônica E A Função Sexual Feminina. Coluna/Columna 19 (4).

Silva et al. (2017). Massagem Perineal Melhora A Dispareunia Causada Por Tensão Dos Músculos Do Assoalho Pélvico. Rev. Bras. Ginecol. Obstet. 39 (1).

Ponzoni et al. (2019). Tratamento Manipulativo Osteopático Musculoesquelético Em Mulheres Com Incontinência Urinária Não Complicada. Fisioterapia Brasil v20n2.

Costa et al. (2018). Cuidado Fisioterapêutico Na Função Sexual Feminina: Intervenção Educativa Na Musculatura Do Assoalho Pélvico. Fisioterapia Brasil v19n1.

Silva et al. (2017). Perfil Clínico E Cinético-Funcional De Puérperas Em Período Imediato. UFS.

Rodrigues et al. (2021). A Influência Da Fisioterapia Na Recuperação Funcional De Pacientes Em Tratamento Do Câncer De Mama. UNISOCIESC.

Rodrigues et al. (2019). Proposta De Uma Ficha De Avaliação Fisioterapêutica Para Pacientes Mastectomizadas. UFU.

Souza et al. (2016). Avaliação Da Força Muscular Do Assoalho Pélvico Em Profissionais Do Sexo Na Cidade De Fortaleza/Ce. Fisioterapia Brasil. 17(6).

Camilo et al. (2019). Alterações Sexuais No Climatério Do Ponto De Vista Cinesiológico-Funcional: Revisão. Rev pesquisa em Fisioterapia. 9(4).

Latorre. (2020). Validação Da Escala Curta De Avaliação Funcional Do Desejo Sexual Feminino. Rev pesquisa em Fisioterapia. 10(1).

Pavanello, D. D. \& Dreher, D. Z. (2020). Fisioterapia na Anorgasmia Feminina: Uma Revisão Integrativa. UNIJUÍ.

Xavier, P. F. P. \& Trindade, A. P. N. T. (2018). Avaliação Do Risco De Queda E Equilíbrio Em Mulheres No Climatério. Rev kairós gerontologia. 21(2). 